A N N A LES

UNIVERSITATIS MARIAE CURIE-SKŁODOWSKA

LUBLIN - POLONIA

\title{
ŁUKASZ PIASECKI
}

\section{On $\ell_{1}$-preduals distant by 1}

\begin{abstract}
For every predual $X$ of $\ell_{1}$ such that the standard basis in $\ell_{1}$ is weak $^{*}$ convergent, we give explicit models of all Banach spaces $Y$ for which the Banach-Mazur distance $d(X, Y)=1$. As a by-product of our considerations, we obtain some new results in metric fixed point theory. First, we show that the space $\ell_{1}$, with a predual $X$ as above, has the stable weak* fixed point property if and only if it has almost stable weak* fixed point property, i.e. the dual $Y^{*}$ of every Banach space $Y$ has the weak* fixed point property (briefly, $\sigma\left(Y^{*}, Y\right)$-FPP) whenever $d(X, Y)=1$. Then, we construct a predual $X$ of $\ell_{1}$ for which $\ell_{1}$ lacks the stable $\sigma\left(\ell_{1}, X\right)$-FPP but it has almost stable $\sigma\left(\ell_{1}, X\right)$-FPP, which in turn is a strictly stronger property than the $\sigma\left(\ell_{1}, X\right)$ FPP. Finally, in the general setting of preduals of $\ell_{1}$, we give a sufficient condition for almost stable weak ${ }^{*}$ fixed point property in $\ell_{1}$ and we prove that for a wide class of spaces this condition is also necessary.
\end{abstract}

1. Introduction and Preliminaries. The notion of nearly isometric Banach spaces was introduced by Stefan Banach in the celebrated Théorie des opérations linéaires [2]. Recall that two isomorphic Banach spaces $X$ and $Y$ are said to be nearly isometric (or almost isometric) if

$$
d(X, Y)=\inf \left\{\|\phi\|\left\|\phi^{-1}\right\|: \phi \text { is an isomorphism from } X \text { onto } Y\right\}=1 .
$$

2010 Mathematics Subject Classification. 46B03, 46B04, 46B20, 46B25, 46B45, 47H09, $47 \mathrm{H} 10$.

Key words and phrases. Banach-Mazur distance, nearly (almost) isometric Banach spaces, $\ell_{1}$-preduals, hyperplanes in $c$, weak ${ }^{*}$ fixed point property, stable weak ${ }^{*}$ fixed point property, almost stable weak* fixed point property, nonexpansive mappings, direct sum, pseudo-metric. 
The constant $d(X, Y)$ is currently named the Banach-Mazur distance between $X$ and $Y$. It is well known that for any finite-dimensional spaces $X$ and $Y, d(X, Y)=1$ if and only if $X$ is isometrically isomorphic to $Y$. Stefan Banach posed the question whether the space $c$ of convergent sequences with the standard sup norm and its subspace $c_{0}$ of sequences convergent to 0 are nearly isometric. A precise answer to Banach's question was given by Michael Cambern in [3], where it is proved that $d\left(c, c_{0}\right)=3$. The first example of two nearly isometric spaces that are non-isometric was given by Czesław Bessaga and Aleksander Pełczyński in [13]. The problem of indicating geometric properties that are not invariant under the Banach-Mazur distance 1 has been posed and studied in [14]. Recall that Property $\mathcal{P}$ is said to be not invariant (or not preserved) under the Banach-Mazur distance 1 if there exist two Banach spaces $X$ and $Y$ such that $X$ has Property $\mathcal{P}, Y$ fails Property $\mathcal{P}$ and $d(X, Y)=1$. On the other hand, we say that Property $\mathcal{P}$ is invariant (or preserved) under the Banach-Mazur distance 1 if for every pair $X, Y$ of Banach spaces with $d(X, Y)=1, X$ has Property $\mathcal{P}$ if and only if $Y$ has Property $\mathcal{P}$. In [14] the reader can find a collection of geometric properties that are invariant under isometries but not invariant under the Banach-Mazur distance 1, even in the restricted setting of preduals of $\ell_{1}$.

In this context, the following problem arises: for a given Banach space $X$, give explicit models of all Banach spaces that are nearly isometric to $X$.

For example, if $X=\ell_{2}$, then every Banach space $Y$ which is almost isometric to $\ell_{2}$ must be isometric to $\ell_{2}$. This is a consequence of the observation that the Parallelogram Law is invariant under the Banach-Mazur distance 1 .

In the present paper we solve this problem for every predual of $\ell_{1}$ such that the standard basis in $\ell_{1}$ is weak* convergent (see Theorem 2.1 and Remark 2.9). This is the main result of our paper and in order to prove it, we shall need some intermediate steps that are interesting in themselves (see Section 2).

In Section 3 we introduce a new property that we call almost stable weak* fixed point property. Then, basing on the results obtained in Section 2, we give examples showing that this property lies strictly between the weak* fixed point property and the stable weak* fixed point property (see Examples $3.4-3.5$ ). It is worth noticing that it is impossible to find such an example if we restrict our attention to the class of preduals of $\ell_{1}$ for which the standard basis in $\ell_{1}$ is weak ${ }^{*}$ convergent. Indeed, in this special case almost stable weak* fixed point property is equivalent to the stable weak* fixed point property (see Proposition 3.1). Moreover, for every predual $X$ of $\ell_{1}$ such that the standard basis in $\ell_{1}$ is weak* convergent and $\ell_{1}$ has the weak* fixed point property but lacks the stable weak* fixed point property, there are infinitely many mutually non-isometric spaces distant from $X$ by 1 whose duals fail the weak* fixed point property (see Remark 3.2). 
Finally, in the general case of preduals of $\ell_{1}$, we give a sufficient condition for almost stable weak* fixed point property in $\ell_{1}$ (see Proposition 3.6) and we prove that for a wide class of spaces this condition is also necessary (see Proposition 3.3, Proposition 3.8 and Remark 3.9). Furthermore, we show that the sufficient condition introduced in Proposition 3.6 is invariant under the Banach-Mazur distance 1 (see Proposition 3.10).

Throughout the paper we use standard terminology and notations. In particular, given a real infinite-dimensional Banach space $X, B_{X}$ and $S_{X}$ denote its closed unit ball and unit sphere, respectively. If $A \subset X$, then ext $A$ and int $A$ stand for the set of all extreme points of $A$ and the interior of $A$, respectively. The dual of $X$ is denoted by $X^{*}$. If $A \subset X^{*}$, then $\bar{A}^{*}$ denotes the $w^{*}$-closure of $A$, and $A^{\prime}$ stands for the set of all $w^{*}$-cluster points of $A$ :

$$
A^{\prime}=\left\{x^{*} \in X^{*}: x^{*} \in{\overline{\left(A \backslash\left\{x^{*}\right\}\right)}}^{*}\right\} \text {. }
$$

For any Banach spaces $X$ and $Y, X=Y$ means that $X$ is isometrically isomorphic to $Y$. Similarly, $X \subset Y$ means that $Y$ contains a subspace which is isometrically isomorphic to $X$. Recall that a Banach space $X$ is called an $L_{1}$-predual or a Lindenstrauss space if $X^{*}=L_{1}(\mu)$ for some measure $\mu$. In particular, $X$ is named an $\ell_{1}$-predual if $X^{*}=\ell_{1}$. The spaces $c_{0}$ and $c$ are among the best-known examples of $\ell_{1}$-preduals.

In the sequel, $\left(e_{n}^{*}\right)$ denotes the standard basis in $\ell_{1}$.

The first main tool in our considerations is a class of hyperplanes in $c$ : for every functional $f=(f(1), f(2), \ldots) \in \ell_{1}=c^{*}$ with $\|f\|=1$ and $f(1) \geq \frac{1}{2}$ we define a hyperplane $W_{f}$ in $c$ by

$$
\begin{aligned}
W_{f} & =\{x \in c: f(x)=0\} \\
& =\left\{x=(x(1), x(2), \ldots) \in c: f(1) \lim _{i \rightarrow \infty} x(i)+\sum_{i=1}^{\infty} f(i+1) x(i)=0\right\} .
\end{aligned}
$$

This class of spaces was widely studied in [4]-[8] and [14]. By Theorem 4.3 in [4], every element $y=(y(1), y(2), \ldots) \in \ell_{1}$ can be identified with a functional $\phi(y) \in W_{f}^{*}$ via the relation

$$
(\phi(y))(x)=\sum_{j=1}^{\infty} x(j) y(j)
$$

for every $x=(x(1), x(2), \ldots) \in W_{f}$, the mapping $\phi: \ell_{1} \rightarrow W_{f}^{*}$ is an isometrical isomorphism and

$$
e_{n}^{*} \stackrel{\sigma\left(\ell_{1}, W_{f}\right)}{\longrightarrow} e^{*}=\left(-\frac{f(2)}{f(1)},-\frac{f(3)}{f(1)},-\frac{f(4)}{f(1)}, \ldots\right) .
$$

(Note that in [6]-[8] and [14] the hyperplane $W_{f}$ is denoted by $W_{e^{*}}$.) This result has some important consequences. Namely, let $e^{*}=\left(e^{*}(1), e^{*}(2), \ldots\right) \in$ 
$B_{\ell_{1}}$ be arbitrarily chosen. Then, for the hyperplane $W_{f}$ with

$$
f=\left(\frac{1}{1+\left\|e^{*}\right\|}, \frac{-e^{*}(1)}{1+\left\|e^{*}\right\|}, \frac{-e^{*}(2)}{1+\left\|e^{*}\right\|}, \frac{-e^{*}(3)}{1+\left\|e^{*}\right\|}, \ldots\right) \in S_{\ell_{1}},
$$

we have $e_{n}^{*} \stackrel{\sigma\left(\ell_{1}, W_{f}\right)}{\longrightarrow} e^{*}$. Moreover, by Corollary 4.4 in [4], if $X$ is a predual of $\ell_{1}$ such that $\left(e_{n}^{*}\right)$ is $\sigma\left(\ell_{1}, X\right)$-convergent to $e^{*}$, then $X$ must be isometrically isomorphic to the hyperplane $W_{f}$.

The second main tool is the so-called property $\left(a^{\infty}\right)$ introduced by E. Michael and A. Pełczyński in [11]. We say that a Banach space $X$ has property $\left(a^{\infty}\right)$ if for every finite set $Z$ in $X$ and for every $\eta>0$, there is an integer $n$ and a linear map $T: \ell_{\infty}^{n} \rightarrow X$ such that

$$
\operatorname{dist}\left(z, T\left(\ell_{\infty}^{n}\right)\right):=\inf _{x \in T\left(\ell_{\infty}^{n}\right)}\|x-z\|<\eta
$$

for every $z \in Z$, and

$$
(1+\eta)^{-1}\|x\| \leq\|T x\| \leq(1+\eta)\|x\|
$$

for every $x \in \ell_{\infty}^{n}$, where $\ell_{\infty}^{n}$ denotes the space of $n$-tuples of reals with the norm $\|x\|=\max _{1 \leq i \leq n}|x(i)|$.

The third main and new tool is the function $p: \ell_{1} \times \ell_{1} \rightarrow[0, \infty)$ defined for every $(x, y) \in \ell_{1} \times \ell_{1}$ by

$$
p(x, y)=\inf \left\{\sum_{n=1}^{\infty}|x(n)-\epsilon(n) y(\pi(n))|\right\},
$$

where the infimum is taken over all permutations $\pi: \mathbb{N} \rightarrow \mathbb{N}$ and all sequences of signs $\epsilon=(\epsilon(n))_{n \in \mathbb{N}}, \epsilon(n)= \pm 1$ for all $n \in \mathbb{N}$.

Below we list some basic properties of $p$, useful in the sequel of this paper:

- $p(x, y)=p(y, x)=p(x,-y)=p(-x,-y)$ for all $x, y \in \ell_{1}$,

- $p(x, y) \leq p(x, z)+p(z, y)$ for all $x, y, z \in \ell_{1}$.

Consequently, $p$ is a pseudo-metric on $\ell_{1}$. However, it is not a metric.

- Let $x=(x(1), x(2), \ldots) \in \ell_{1}$. Let $\mathcal{E}$ be the set of all sequences of signs $\epsilon=(\epsilon(n))_{n \in \mathbb{N}}, \epsilon(n)= \pm 1$ for all $n \in \mathbb{N}$. For $\epsilon \in \mathcal{E}$ and $i \in \mathbb{N} \cup\{0\}$ we define

$$
x_{i, \epsilon}=\sum_{n=1}^{\infty} \epsilon(n) x(n) e_{n+i}^{*}
$$

and for $i=\infty$ we put

$$
x_{\infty, \epsilon}=\sum_{n=1}^{\infty} \epsilon(n) x(n) e_{2 n-1}^{*} .
$$


Let $\Pi$ be the set of all permutations $\pi: \mathbb{N} \rightarrow \mathbb{N}$. Then, for $\epsilon \in \mathcal{E}$, $\pi \in \Pi$ and $i \in \mathbb{N} \cup\{0, \infty\}$ we define

$$
x_{i, \epsilon, \pi}=\sum_{n=1}^{\infty} x_{i, \epsilon}(n) e_{\pi(n)}^{*} \text {. }
$$

Let

$$
\widetilde{\mathcal{F}_{x}}=\left\{x_{i, \epsilon, \pi}: i \in \mathbb{N} \cup\{0, \infty\}, \epsilon \in \mathcal{E}, \pi \in \Pi\right\} .
$$

It is easy to see that for any $x, y \in \ell_{1}, p(x, y)=0$ if and only if $y \in \widetilde{\mathcal{F}}_{x}$.

We also have the following relation between the function $p$ and the standard norm in $\ell_{1}$ :

- for all $x, y \in \ell_{1}$,

$$
|\|x\|-\|y\|| \leq p(x, y) \leq\|x-y\| .
$$

2. Main results. Let $f \in S_{\ell_{1}}$ be such that $f(1) \geq \frac{1}{2}$. For $\epsilon \in \mathcal{E}$ and $i \in \mathbb{N} \cup\{0\}$ we define

$$
f_{i, \epsilon}=f(1) e_{1}^{*}+\sum_{n=1}^{\infty} \epsilon(n) f(n+1) e_{n+i+1}^{*}
$$

and for $i=\infty$ we put

$$
f_{\infty, \epsilon}=f(1) e_{1}^{*}+\sum_{n=1}^{\infty} \epsilon(n) f(n+1) e_{2 n+1}^{*}
$$

Let

$$
\mathcal{F}_{W_{f}}=\left\{W_{f_{i, \epsilon}}: i \in \mathbb{N} \cup\{0, \infty\}, \epsilon \in \mathcal{E}\right\} .
$$

In what follows, $X \in \mathcal{F}_{W_{f}}$ means that a Banach space $X$ is isometrically isomorphic to $W_{f_{i}, \epsilon}$ for some $i \in \mathbb{N} \cup\{0, \infty\}$ and for some $\epsilon \in \mathcal{E}$.

We are ready now to state our main result.

Theorem 2.1. Let $f \in S_{\ell_{1}}$ be such that $f(1) \geq \frac{1}{2}$ and let $X$ be a Banach space. Then the following are equivalent.

(1) $d\left(X, W_{f}\right)=1$.

(2) $X \in \mathcal{F}_{W_{f}}$.

The proof of the implication $(1) \Rightarrow(2)$ in Theorem 2.1 follows from the series of results given below. We begin by proving Proposition 2.2 and Lemmas 2.3-2.4, since it will allow to restrict our considerations to the case of hyperplanes in $c$.

Proposition 2.2. The property of being a Lindenstrauss space is invariant under the Banach-Mazur distance 1. In particular, for all Banach spaces $X$ and $Y$ with $d(X, Y)=1, X$ is a predual of $\ell_{1}$ if and only if $Y$ is a predual of $\ell_{1}$. 
Proof. It is straightforward to check that the property $\left(a^{\infty}\right)$ is invariant under the Banach-Mazur distance 1. Our assertion follows now from Theorem 1 in [12].

Lemma 2.3. Let $f \in S_{\ell_{1}}$ be such that $f(1) \geq \frac{1}{2}$ and let $X$ be a Banach space. If $d\left(X, W_{f}\right)=1$, then (ext $\left.B_{X^{*}}\right)^{\prime}=\left\{ \pm x^{*}\right\}$ for some $x^{*} \in B_{X^{*}}$, that is, $\left(\text { ext } B_{X^{*}}\right)^{\prime}$ is either a singleton or consists of two elements.

Proof. By Proposition 2.2, $X$ is a predual of $\ell_{1}$. Since $d\left(X, W_{f}\right)=1$, for every $m \in \mathbb{N}$ there exists an adjoint isomorphism $\phi_{m}^{*}: X^{*} \rightarrow W_{f}^{*}$ such that for every $n \in \mathbb{N}$

$$
\phi_{m}^{*}\left(e_{n}^{*}\right)=\epsilon(n) e_{\pi(n)}^{*}+w_{n}^{*},
$$

where $\pi: \mathbb{N} \rightarrow \mathbb{N}$ is a permutation, $\epsilon=(\epsilon(n))_{n \in \mathbb{N}}$ is a sequence of signs and $\left\|w_{n}^{*}\right\|<\frac{1}{m}$ for all $n \in \mathbb{N}$. Using the mappings $\phi_{m}^{*}$, we can easily see that if $f=(1,0,0, \ldots)$, then $\left(\operatorname{ext} B_{X^{*}}\right)^{\prime}=\{0\}$, and if $f \neq(1,0,0, \ldots)$, then $\left(\text { ext } B_{X^{*}}\right)^{\prime}$ has exactly two elements.

Lemma 2.4. Let $X$ be a predual of $\ell_{1}$ such that $\left(\text { ext } B_{X^{*}}\right)^{\prime}=\left\{ \pm x^{*}\right\}$, that is, $\left(\text { ext } B_{X^{*}}\right)^{\prime}$ is either a singleton or consists of two elements. Then $X$ is isometric to some hyperplane $W_{f}$.

Proof. Since $B_{\ell_{1}}$ is $\sigma\left(\ell_{1}, X\right)$-compact and the $\sigma\left(\ell_{1}, X\right)$-topology is metrizable on $B_{\ell_{1}}$, we can choose a sequence of signs $\epsilon=(\epsilon(n))_{n \in \mathbb{N}}$ such that the sequence $\left(\epsilon(n) e_{n}^{*}\right)_{n \in \mathbb{N}}$ is $\sigma\left(\ell_{1}, X\right)$-convergent to $x^{*}$. Consider the hyperplane $W_{f}$ with

$$
f=\left(\frac{1}{1+\left\|x^{*}\right\|},-\frac{\epsilon(1) x^{*}(1)}{1+\left\|x^{*}\right\|},-\frac{\epsilon(2) x^{*}(2)}{1+\left\|x^{*}\right\|},-\frac{\epsilon(3) x^{*}(3)}{1+\left\|x^{*}\right\|}, \ldots\right) .
$$

Then

$$
e_{n}^{*} \stackrel{\sigma\left(\ell_{1}, W_{f}\right)}{\longrightarrow} e^{*}=\left(\epsilon(1) x^{*}(1), \epsilon(2) x^{*}(2), \epsilon(3) x^{*}(3), \ldots\right) .
$$

Let $\phi^{*}: X^{*} \rightarrow W_{f}^{*}$ be defined by

$$
\phi^{*}(z)=\sum_{n=1}^{\infty} z^{*}(n) \epsilon(n) e_{n}^{*} .
$$

$\phi^{*}$ is an onto linear isometry. Since $\phi^{*}\left(x^{*}\right)=e^{*}, \phi^{*}$ is a $w^{*}$-continuous homeomorphism from $\overline{\left\{\epsilon(n) e_{n}^{*}: n \in \mathbb{N}^{*}\right.}=\left\{\epsilon(n) e_{n}^{*}: n \in \mathbb{N}\right\} \cup\left\{x^{*}\right\}$ onto ${\overline{\left\{e_{n}^{*}: n \in \mathbb{N}\right\}}}^{*}=\left\{e_{n}^{*}: n \in \mathbb{N}\right\} \cup\left\{e^{*}\right\}$. By Lemma 2 in [1], $\phi^{*}$ is a $w^{*}$ continuous isometry from $X^{*}$ onto $W_{f}^{*}$. This shows that $\phi^{*}$ must be the adjoint to an isometry $\phi: W_{f} \rightarrow X$.

The following result gives a necessary condition for any two $\ell_{1}$-preduals to be Banach-Mazur distance 1 apart. 
Proposition 2.5. Let $X$ and $Y$ be preduals of $\ell_{1}$ with $d(X, Y)=1$. Then for every $y^{*} \in\left(\text { ext } B_{Y^{*}}\right)^{\prime}$ there exists a sequence $\left(x_{m}^{*}\right) \subset\left(\text { ext } B_{X^{*}}\right)^{\prime}$ such that

$$
\lim _{m \rightarrow \infty} p\left(x_{m}^{*}, y^{*}\right)=0
$$

Proof. As in the proof of Lemma 2.3, since $d(X, Y)=1$, for every $m \in \mathbb{N}$ there exists an adjoint isomorphism $\phi_{m}^{*}: Y^{*} \rightarrow X^{*}$ such that for every $n \in \mathbb{N}$

$$
\phi_{m}^{*}\left(e_{n}^{*}\right)=\epsilon(n) e_{\pi(n)}^{*}+w_{n}^{*},
$$

where $\pi: \mathbb{N} \rightarrow \mathbb{N}$ is a permutation, $\epsilon=(\epsilon(n))_{n \in \mathbb{N}}$ is a sequence of signs and $\left\|w_{n}^{*}\right\|<\frac{1}{m}$ for all $n \in \mathbb{N}$. Without loss of generality we can assume that there is a subsequence $\left(e_{n_{k}}^{*}\right)$ of $\left(e_{n}^{*}\right)$ such that $e_{n_{k}}^{*} \stackrel{\sigma\left(\ell_{1}, Y\right)}{\longrightarrow} y^{*}$. Let $x_{m}^{*} \in X^{*}$ be a cluster point of $\left(\epsilon\left(n_{k}\right) e_{\pi\left(n_{k}\right)}^{*}\right)_{k \in \mathbb{N}}$. By passing to a subsequence, if necessary, we can assume that $\epsilon\left(n_{k}\right) e_{\pi\left(n_{k}\right)}^{*} \stackrel{\sigma\left(\ell_{1}, X\right)}{\longrightarrow} x_{m}^{*}$. Then

$$
\begin{aligned}
p\left(x_{m}^{*}, y^{*}\right) & \leq \sum_{n=1}^{\infty}\left|x_{m}^{*}(n)-\epsilon\left(\pi^{-1}(n)\right) y^{*}\left(\pi^{-1}(n)\right)\right| \\
& \leq \sum_{n=1}^{\infty}\left|x_{m}^{*}(n)-\left(\phi_{m}^{*}\left(y^{*}\right)\right)(n)\right|+\sum_{n=1}^{\infty} \sum_{j=1}^{\infty}\left|w_{j}^{*}(n)\right|\left|y^{*}(j)\right| \\
& \leq\left\|x_{m}^{*}-\phi_{m}^{*}\left(y^{*}\right)\right\|+\frac{1}{m} \\
& \leq \liminf _{k \rightarrow \infty}\left\|\epsilon\left(n_{k}\right) e_{\pi\left(n_{k}\right)}^{*}-\phi_{m}^{*}\left(e_{n_{k}}^{*}\right)\right\|+\frac{1}{m} \leq \frac{2}{m} .
\end{aligned}
$$

Now it is enough to pass to the limit with $m \rightarrow \infty$.

In the next result of this section, for every hyperplane $W_{f}$ we describe all the hyperplanes $W_{g}$ that are isometrically isomorphic to $W_{f}$. In particular, it allows us to finish the proof of the implication $(1) \Rightarrow(2)$ in Theorem 2.1.

Proposition 2.6. For $f, g \in S_{\ell_{1}}$ with $f(1) \geq \frac{1}{2}$ and $g(1) \geq \frac{1}{2}$ the following statements are equivalent.

(1) $W_{f}=W_{g}$.

(2) There is a finite sequence of signs $(\epsilon(n))_{n=1}^{j}, \epsilon(n)= \pm 1$ for all $n=1, \ldots, j$, and a permutation $\pi: \mathbb{N} \backslash\{1\} \rightarrow \mathbb{N} \backslash\{1\}$ such that

$$
f=g(1) e_{1}^{*}+\sum_{n=1}^{j} \epsilon(n) g(n+1) e_{\pi(n+1)}^{*}+\sum_{n=j+1}^{\infty} g(n+1) e_{\pi(n+1)}^{*} .
$$

Proof. $(2) \Rightarrow(1)$. An isometrical isomorphism $\phi: W_{g} \rightarrow W_{f}$ is given for every $x \in W_{g}$ by 


$$
(\phi(x))(\pi(n+1)-1)= \begin{cases}\epsilon(n) x(n) & \text { for } n=1, \ldots, j \\ x(n) & \text { for } n>j .\end{cases}
$$

$(1) \Rightarrow(2)$. We know that

$$
e_{n}^{*} \stackrel{\sigma\left(\ell_{1}, W_{f}\right)}{\longrightarrow}\left(-\frac{f(2)}{f(1)},-\frac{f(3)}{f(1)}, \ldots\right)=x^{*}
$$

and

$$
e_{n}^{*} \stackrel{\sigma\left(\ell_{1}, W_{g}\right)}{\longrightarrow}\left(-\frac{g(2)}{g(1)},-\frac{g(3)}{g(1)}, \ldots\right)=y^{*} .
$$

Consequently, $f(1)=1$ if and only if $g(1)=1$. Suppose that $f(1) \in$ $[1 / 2,1)$. Let $\phi: W_{f} \rightarrow W_{g}$ be an isometrical isomorphism. Then also its adjoint $\phi^{*}: W_{g}^{*} \rightarrow W_{f}^{*}$ is an isometrical isomorphism. Therefore, there exist a permutation $\pi^{\prime}: \mathbb{N} \rightarrow \mathbb{N}$ and a sequence of signs $\epsilon=(\epsilon(n))_{n \in \mathbb{N}}$ such that $\phi^{*}\left(e_{n}^{*}\right)=\epsilon(n) e_{\pi^{\prime}(n)}^{*}$. Since $\phi^{*}$ is $w^{*}$-continuous and $\left(e_{n}^{*}\right)$ is $\sigma\left(\ell_{1}, W_{g}\right)$ convergent, the sequence $\left(\phi^{*}\left(e_{n}^{*}\right)\right)$ is $\sigma\left(\ell_{1}, W_{f}\right)$-convergent. Consequently, there exists $j \in \mathbb{N}$ such that either $\epsilon(n)=1$ for all $n>j$ or $\epsilon(n)=-1$ for all $n>j$. Suppose that $\epsilon(n)=1$ for all $n>j$ (otherwise consider $-\phi$ ). Again, by the $w^{*}$-continuity of $\phi^{*}$, we have

$$
\begin{aligned}
x^{*} & =\phi^{*}\left(y^{*}\right)=\sum_{n=1}^{\infty} y^{*}(n) \epsilon(n) e_{\pi^{\prime}(n)}^{*} \\
& =-\sum_{n=1}^{j} \frac{g(n+1)}{g(1)} \epsilon(n) e_{\pi^{\prime}(n)}^{*}-\sum_{n=j+1}^{\infty} \frac{g(n+1)}{g(1)} e_{\pi^{\prime}(n)}^{*} .
\end{aligned}
$$

Taking into account that $f(1) \geq \frac{1}{2}, g(1) \geq \frac{1}{2}$ and $\|f\|=\|g\|=1$, we get $f(1)=g(1)$ and so

$$
\sum_{n=1}^{\infty} f(n+1) e_{n}^{*}=\sum_{n=1}^{j} g(n+1) \epsilon(n) e_{\pi^{\prime}(n)}^{*}+\sum_{n=j+1}^{\infty} g(n+1) e_{\pi^{\prime}(n)}^{*} .
$$

Finally, by putting $\pi(n+1)=\pi^{\prime}(n)+1$ for all $n \in \mathbb{N}$, we get

$$
f=\sum_{n=1}^{\infty} f(n) e_{n}^{*}=g(1) e_{1}^{*}+\sum_{n=1}^{j} g(n+1) \epsilon(n) e_{\pi(n+1)}^{*}+\sum_{n=j+1}^{\infty} g(n+1) e_{\pi(n+1)}^{*} .
$$

Corollary 2.7. Let $X$ and $Y$ be preduals of $\ell_{1}$ such that $\left(e_{n}^{*}\right)$ is $\sigma\left(\ell_{1}, X\right)$ convergent to $x^{*}$ and $\sigma\left(\ell_{1}, Y\right)$-convergent to $y^{*}$. Then the following are equivalent.

(1) $X=Y$. 
(2) There is a finite sequence of signs $(\epsilon(n))_{n=1}^{j}, \epsilon(n)= \pm 1$ for all $n=1, \ldots, j$, and a permutation $\pi: \mathbb{N} \rightarrow \mathbb{N}$ such that

$$
x^{*}=\sum_{n=1}^{j} \epsilon(n) y^{*}(n) e_{\pi(n)}^{*}+\sum_{n=j+1}^{\infty} y^{*}(n) e_{\pi(n)}^{*} .
$$

We shall prove now the implication $(2) \Rightarrow(1)$ in Theorem 2.1:

Proof. $(2) \Rightarrow(1)$. For $\epsilon \in \mathcal{E}$ and $j \in \mathbb{N}$ we define

$$
f_{0, \epsilon}^{(j)}=f(1) e_{1}^{*}+\sum_{n=1}^{j} f(n+1) e_{n+1}^{*}+\sum_{n=j+1}^{\infty} \epsilon(n) f(n+1) e_{n+1}^{*} .
$$

By Lemma 2.1 in [6] and Proposition 2.6, we get

$$
d\left(W_{f_{0, \epsilon}}, W_{f}\right) \leq d\left(W_{f_{0, \epsilon}}, W_{f_{0, \epsilon}}^{(j)}\right) \cdot d\left(W_{f_{0, \epsilon}}^{(j)}, W_{f}\right)=d\left(W_{f_{0, \epsilon}}^{(j)}, W_{f}\right) \stackrel{j \rightarrow \infty}{\longrightarrow} 1 .
$$

Therefore $d\left(W_{f_{0, \epsilon}}, W_{f}\right)=1$. Next, for $i \in \mathbb{N}, \epsilon \in \mathcal{E}$ and $j \in \mathbb{N}$ we put

$$
f_{i, \epsilon}^{(j)}=f(1) e_{1}^{*}+\sum_{n=1}^{j} \epsilon(n) f(n+1) e_{n+1}^{*}+\sum_{n=j+1}^{\infty} \epsilon(n) f(n+1) e_{n+i+1}^{*} .
$$

Again, by Lemma 2.1 in [6] and Proposition 2.6, we get $d\left(W_{f_{0, \epsilon}}, W_{f_{i, \epsilon}}\right)=1$. Finally, in order to prove that $d\left(W_{f_{\infty, \epsilon}}, W_{f_{0, \epsilon}}\right)=1$, it is enough to repeat the above reasoning with

$$
f_{\infty, \epsilon}^{(j)}=f(1) e_{1}^{*}+\sum_{n=1}^{j} \epsilon(n) f(n+1) e_{n+1}^{*}+\sum_{n=j+1}^{\infty} \epsilon(n) f(n+1) e_{2 n+1}^{*} .
$$

Consequently, $d\left(X, W_{f}\right)=1$ for every $X \in \mathcal{F}_{W_{f}}$.

From Theorem 2.1, Proposition 2.6, and Corollary 2.7 we obtain the following

Corollary 2.8. Let $f, g \in S_{\ell_{1}}$ be such that $f(1) \geq \frac{1}{2}$ and $g(1) \geq \frac{1}{2}$. Then the following are equivalent.

(1) $d\left(W_{f}, W_{g}\right)=1$.

(2) $p(f, g)=0$.

(3) $p\left(x^{*}, y^{*}\right)=0$, where $e_{n}^{*} \stackrel{\sigma\left(\ell_{1}, W_{f}\right)}{\longrightarrow} x^{*}$ and $e_{n}^{*} \stackrel{\sigma\left(\ell_{1}, W_{g}\right)}{\longrightarrow} y^{*}$.

Remark 2.9. We finish this section with yet another observation resulting from Theorem 2.1 and Proposition 2.6. Namely, for every hyperplane $W_{f}$ we have one of two possibilities: either there is exactly one Banach space that is distant from $W_{f}$ by 1 (equivalently, $f=(f(1), \ldots, f(n), 0,0, \ldots)$ for some $n \in \mathbb{N}$ ) or there are infinitely many mutually non-isometric spaces that are distant from $W_{f}$ by 1 (equivalently, $f(n) \neq 0$ for infinitely many $n \in \mathbb{N}$ ). Consequently, for a given $f \in S_{\ell_{1}}$ with $f(1) \geq 1 / 2$, the property of 
being the hyperplane $W_{f}$ is invariant under the Banach-Mazur distance 1 if and only if $f=(f(1), \ldots, f(n), 0,0, \ldots)$ for some $n \in \mathbb{N}$. In particular, this holds for the spaces $c_{0}$ and $c$.

3. Almost stable weak* fixed point property. We shall present now some applications of our results in metric fixed point theory. The reader who is not familiar with this topic is referred to the book [9].

A nonempty, bounded, closed and convex subset $C$ of $X$ has the fixed point property (briefly, FPP) if each nonexpansive mapping (i.e., the mapping $T: C \rightarrow C$ such that $\|T(x)-T(y)\| \leq\|x-y\|$ for all $x, y \in C$ ) has a fixed point. A dual space $X^{*}$ is said to have the weak fixed point property (briefly, $w^{*}$-FPP or $\sigma\left(X^{*}, X\right)$-FPP) if every nonempty, convex, $\sigma\left(X^{*}, X\right)$-compact set $C \subset X^{*}$ has the FPP. Moreover, $X^{*}$ is said to have the stable weak fixed point property (briefly, stable $w^{*}$-FPP or stable $\sigma\left(X^{*}, X\right)$-FPP) if there exists $\gamma>1$ such that $Y^{*}$ has the $\sigma\left(Y^{*}, Y\right)$-FPP whenever $d(X, Y)<\gamma$.

We introduce now a new definition related to the $\sigma\left(X^{*}, X\right)$-FPP: we will say that $X^{*}$ has almost stable weak* fixed point property (briefly, almost stable $w^{*}$-FPP or almost stable $\sigma\left(X^{*}, X\right)$-FPP) if $Y^{*}$ has the $\sigma\left(Y^{*}, Y\right)$ FPP whenever $d(X, Y)=1$.

Clearly, for every dual space $X^{*}$,

$$
\text { stable } w^{*} \text {-FPP } \Rightarrow \text { almost stable } w^{*} \text {-FPP } \Rightarrow w^{*} \text {-FPP. }
$$

However, the reversed implications do not hold in general. Indeed, Examples $3.2-3.3$ in [14] show that

$$
w^{*} \text {-FPP } \nRightarrow \text { almost stable } w^{*} \text {-FPP }
$$

and Examples 3.4-3.5 presented below prove that

$$
\text { almost stable } w^{*} \text {-FPP } \nRightarrow \text { stable } w^{*} \text {-FPP. }
$$

However, we begin by proving that for every predual of $\ell_{1}$ such that $\left(e_{n}^{*}\right)$ is weak* convergent, almost stable weak* fixed point property and the stable weak* fixed point property are equivalent.

Proposition 3.1. Let $X$ be a predual of $\ell_{1}$ such that $\left(e_{n}^{*}\right)$ is $\sigma\left(\ell_{1}, X\right)$ convergent to $e^{*}$. Then the following are equivalent.

(1) $\ell_{1}$ has almost stable $\sigma\left(\ell_{1}, X\right)-F P P$.

(2) $\ell_{1}$ has the stable $\sigma\left(\ell_{1}, X\right)-F P P$.

(3) $\left\|e^{*}\right\|<1$.

(4) For every $x^{*} \in S_{\ell_{1}}$ we have $p\left(e^{*}, x^{*}\right)>0$.

(5) For every Banach space $Y$ such that $d(X, Y)=1, c \not \subset Y$.

Proof. Clearly, $(2) \Rightarrow(1)$ and $(3) \Leftrightarrow(4)$. The equivalence $(2) \Leftrightarrow(3)$ is a particular case of Theorem 3.5 in [7]. The implication $\neg(5) \Rightarrow \neg(1)$ follows from Theorem 3.2 in [5]. We show that $\neg(3) \Rightarrow \neg(5)$. Suppose that $\left\|e^{*}\right\|=1$. Then $X=W_{f}$, where $f=\left(1 / 2,-e^{*}(1) / 2,-e^{*}(2) / 2, \ldots\right)$. Let $\epsilon=(\epsilon(n))_{n \in \mathbb{N}}$ 
be the sequence of signs with $\epsilon(n)=-\operatorname{sgn}(f(n+1))$ for all $n \in \mathbb{N}$. By Theorem 2.1, $d\left(W_{f}, W_{f_{\infty}, \epsilon}\right)=1$. By Proposition 2.1 in [5], $c \subset W_{f_{\infty}, \epsilon}$.

Remark 3.2. By Theorem 8 in [10] (and Proposition 2.2 in [5]) and Proposition 3.1, the space $\ell_{1}$, with a predual $X$ as above, has the $\sigma\left(\ell_{1}, X\right)$-FPP and, at the same time, lacks the stable $\sigma\left(\ell_{1}, X\right)$-FPP if and only if $X=W_{f}$, where $f(1)=1 / 2$ and the set $N^{+}=\{n \in \mathbb{N}: f(n+1) \leq 0\}$ is finite. From this, Theorem 2.1, and Proposition 2.6, we get that for every such space $W_{f}$ there are infinitely many mutually non-isometric spaces $W_{f_{i, \epsilon}} \in \mathcal{F}_{W_{f}}$ such that $\ell_{1}$ has the $\sigma\left(\ell_{1}, W_{f_{i, \epsilon}}\right)$-FPP and, at the same time, there are infinitely many mutually non-isometric spaces $W_{f_{i, \epsilon}} \in \mathcal{F}_{W_{f}}$ such that $\ell_{1}$ lacks the $\sigma\left(\ell_{1}, W_{f_{i, \epsilon}}\right)$-FPP. Indeed, for every $\epsilon \in \mathcal{E}$ the set $\left\{W_{f_{i, \epsilon}}: i \in \mathbb{N} \cup\{0\}\right\}$ consists of mutually non-isometric spaces such that $d\left(W_{f}, W_{f_{i, \epsilon}}\right)=1$ and $\ell_{1}$ has the $\sigma\left(\ell_{1}, W_{f_{i, \epsilon}}\right)$-FPP for each $i \in \mathbb{N} \cup\{0\}$. On the other hand, for every $k \in \mathbb{N} \cup\{0\}$ consider a sequence of signs $\epsilon_{k}=\left(\epsilon_{k}(n)\right)$ defined by $\epsilon_{k}(n)=(-1)^{\left[(n-1) / 2^{k}\right]}$ for all $n \in \mathbb{N}$, where $[x]:=\max \{m \in \mathbb{Z}: m \leq x\}$. Then the set $\left\{W_{f_{\infty, \epsilon_{k}}}: k \in \mathbb{N} \cup\{0\}\right\}$ consists of mutually non-isometric spaces such that $d\left(W_{f}, W_{f_{\infty, \epsilon_{k}}}\right)=1$ and $\ell_{1}$ fails the $\sigma\left(\ell_{1}, W_{f_{\infty, \epsilon_{k}}}\right)$-FPP for each $k \in \mathbb{N} \cup\{0\}$.

We also have a similar situation for the class of finite direct sums of hyperplanes in $c$ :

Proposition 3.3. Let $\left\{f_{i}\right\}_{i=1}^{m} \subset S_{\ell_{1}}$ be such that $f_{i}(1) \geq 1 / 2$ for all $i=$ $1, \ldots, m$ and let $x_{i}^{*} \in \ell_{1}$ be the $\sigma\left(\ell_{1}, W_{f_{i}}\right)$-limit of $\left(e_{n}^{*}\right)$. Let $X$ be the $\ell_{\infty}^{m}$ direct sum of hyperplanes $W_{f_{i}}, X=\left(\sum_{i=1}^{m} W_{f_{i}}\right)_{\ell_{\infty}^{m}}$. Then the following are equivalent.

(1) $\ell_{1}$ has almost stable $\sigma\left(\ell_{1}, X\right)-F P P$.

(2) $\ell_{1}$ has the stable $\sigma\left(\ell_{1}, X\right)-F P P$.

(3) $\left\|x_{i}^{*}\right\|<1$ for every $i=1, \ldots, m$.

(4) For every $x^{*} \in S_{\ell_{1}}$ and for every $i=1, \ldots, m$ we have $p\left(x_{i}^{*}, x^{*}\right)>0$.

(5) For every Banach space $Y$ such that $d(X, Y)=1, c \not \subset Y$.

Proof. The proof is similar to the proof of Proposition 3.1, so we omit it.

We provide now some examples of $\ell_{1}$-preduals such that $\ell_{1}$ has almost stable weak* fixed point property but it fails the stable weak* fixed point property.

Example 3.4. For $m \in \mathbb{N}$ let

$$
f_{m}=(10^{m} /\left(2 \cdot 10^{m}-1\right), \underbrace{-1 /\left(2 \cdot 10^{m}-1\right), \ldots,-1 /\left(2 \cdot 10^{m}-1\right)}_{10^{m}-1}, 0,0, \ldots) .
$$


Then, for every $m \in \mathbb{N}$,

$$
e_{n}^{*} \stackrel{\sigma\left(\ell_{1}, W_{f_{m}}\right)}{\longrightarrow} x_{m}^{*}=(\underbrace{1 / 10^{m}, \ldots, 1 / 10^{m}}_{10^{m}-1}, 0,0, \ldots) .
$$

We define $X$ as the $c_{0}$-direct sum of hyperplanes $W_{f_{m}}, X=\left(\sum_{m=1}^{\infty} W_{f_{m}}\right)_{c_{0}}$. Then $X^{*}=\left(\sum_{m=1}^{\infty} \ell_{1}\right)_{\ell_{1}}=\ell_{1}$ and

$$
\left(\operatorname{ext} B_{X^{*}}\right)^{\prime}=\{(0,0, \ldots)\} \cup \bigcup_{m=1}^{\infty}\left\{ \pm(\underbrace{0, \ldots, 0}_{m-1}, x_{m}^{*}, 0,0, \ldots)\right\} \subset \operatorname{int} B_{\ell_{1}} .
$$

From this and Theorem 4.1 in [5] we see that $\ell_{1}$ has the $\sigma\left(\ell_{1}, X\right)$-FPP. On the other hand, since $\lim _{m \rightarrow \infty}\left\|x_{m}^{*}\right\|=1, \ell_{1}$ lacks the stable $\sigma\left(\ell_{1}, X\right)$-FPP by Theorem 3.5 in [7]. We show that $\ell_{1}$ has almost stable $\sigma\left(\ell_{1}, X\right)$-FPP. Let $\widetilde{x_{m}^{*}}=(\underbrace{0, \ldots, 0}_{m-1}, x_{m}^{*}, 0,0, \ldots), m \in \mathbb{N}$. For all $m>n$, we have

$$
\begin{aligned}
p\left(\widetilde{x_{n}^{*}}, \widetilde{x_{m}^{*}}\right) & =p\left(-\widetilde{x_{n}^{*}}, \widetilde{x_{m}^{*}}\right)=p\left(x_{n}^{*}, x_{m}^{*}\right)=p\left(-x_{n}^{*}, x_{m}^{*}\right) \\
& =\left(\frac{1}{10^{n}}-\frac{1}{10^{m}}\right)\left(10^{n}-1\right)+\frac{1}{10^{m}}\left(10^{m}-1-10^{n}+1\right)>\frac{17}{10} .
\end{aligned}
$$

Consequently, for every $y^{*} \in S_{\ell_{1}}$ and for every sequence of signs $\epsilon=$ $(\epsilon(m))_{m \in \mathbb{N}}$, we have

$$
\liminf _{m \rightarrow \infty} p\left(\epsilon(m) \widetilde{x_{m}^{*}}, y^{*}\right)>0 .
$$

By using this, Proposition 2.2, and Proposition 2.5, we see that for every Banach space $Y$ such that $d(X, Y)=1$ we have $Y^{*}=\ell_{1}$ and $\left(\text { ext } B_{Y^{*}}\right)^{\prime} \subset$ int $B_{\ell_{1}}$. Therefore, by Theorem 4.1 in [5], $Y^{*}$ has the $\sigma\left(Y^{*}, Y\right)$-FPP.

Our next example shows that there are many spaces $X$ such that $X^{*}$ fails the stable $\sigma\left(X^{*}, X\right)$-FPP but it has almost stable $\sigma\left(X^{*}, X\right)$-FPP, which in turn is a strictly stronger property than the $\sigma\left(X^{*}, X\right)$-FPP.

Example 3.5. Let $X$ be as in Example 3.4. Let $\left(r_{m}\right)_{m \in \mathbb{N}}$ be a sequence of all rational numbers in the interval $[0,1 / 2]$. Put $g_{m}=\left(\frac{1}{1+r_{m}}, \frac{-r_{m}}{1+r_{m}}, 0,0, \ldots\right)$ and define $Y$ as

$$
Y=\left(X \oplus\left(\sum_{m=1}^{\infty} W_{g_{m}}\right)_{c_{0}}\right)_{\infty}
$$

Clearly, $Y^{*}=\ell_{1}$. By following the reasoning from Example 3.4, we see that $\ell_{1}$ has almost stable $\sigma\left(\ell_{1}, Y\right)$-FPP but fails the stable $\sigma\left(\ell_{1}, Y\right)$-FPP. Let $p_{1}$, $p_{2}$ be arbitrarily chosen irrational numbers in $[0,1 / 2], p_{1} \neq p_{2}$. Put

$$
h_{1}=\left(\frac{1}{1+p_{1}}, \frac{-p_{1}}{1+p_{1}}, 0,0, \ldots\right) \quad \text { and } \quad h_{2}=\left(\frac{1}{1+p_{2}}, \frac{-p_{2}}{1+p_{2}}, 0,0, \ldots\right) .
$$


Let $Z_{1}=\left(Y \oplus W_{h_{1}}\right)_{\infty}$ and $Z_{2}=\left(Y \oplus W_{h_{2}}\right)_{\infty}$. By applying Lemma $3.1 \mathrm{in}$ [14] and Lemma 2.1 in [6], we easily see that $d\left(Y, Z_{1}\right)=d\left(Y, Z_{2}\right)=1$. Since $\left(\text { ext } B_{Z_{1}^{*}}\right)^{\prime}$ contains an element with norm $p_{1}$ and $\left(\text { ext } B_{Z_{2}^{*}}\right)^{\prime}$ does not have this property, the spaces $Z_{1}$ and $Z_{2}$ are not isometric. Similarly, $Z_{1} \neq Y$. This shows that there are uncountable many mutually non-isometric spaces that are distant from $Y$ by 1.

We summarize the above considerations in the following:

Proposition 3.6. Let $X$ be a predual of $\ell_{1}$. If for every $x^{*} \in S_{\ell_{1}}$ and for every sequence $\left(x_{n}^{*}\right)$ in $\left(\text { ext } B_{X^{*}}\right)^{\prime}$ we have

$$
\liminf _{n \rightarrow \infty} p\left(x_{n}^{*}, x^{*}\right)>0,
$$

then $\ell_{1}$ has almost stable $\sigma\left(\ell_{1}, X\right)-F P P$.

Proof. It is enough to follow the reasoning from Example 3.4 based on Proposition 2.2 and Proposition 2.5 of the present paper, and Theorem 4.1 in $[5]$.

Remark 3.7. The assumption $(\diamond)$ in Proposition 3.6 can not be replaced by the weaker one: "Let $X$ be a predual of $\ell_{1}$ such that $\left(\text { ext } B_{X^{*}}\right)^{\prime} \subset \operatorname{int} B_{\ell_{1}}$ " (see Example 3.2 in [14]).

Clearly, by Proposition 3.1 and Proposition 3.3, the sufficient condition $(\diamond)$ introduced in Proposition 3.6 becomes also necessary for the class of all finite $\ell_{\infty}^{n}$-direct sums of $\ell_{1}$-predual hyperplanes in $c$. Moreover, the same is true for the class of all $c_{0}$-direct sums:

Proposition 3.8. Let $\left\{f^{(m)}\right\}_{m=1}^{\infty} \subset S_{\ell_{1}}$ be such that $f^{(m)}(1) \geq 1 / 2$ for all $m \in \mathbb{N}$. Let $X$ be the $c_{0}$-direct sum of hyperplanes $W_{f(m)}$,

$$
X=\left(\sum_{m=1}^{\infty} W_{f(m)}\right)_{c_{0}} .
$$

Then the following are equivalent.

(1) $\ell_{1}$ has almost stable $\sigma\left(\ell_{1}, X\right)-F P P$.

(2) For every $x^{*} \in S_{\ell_{1}}$ and for every sequence $\left(x_{n}^{*}\right)$ in $\left(\text { ext } B_{X^{*}}\right)^{\prime}$ we have $\liminf _{n \rightarrow \infty} p\left(x_{n}^{*}, x^{*}\right)>0$ (property $\left.(\diamond)\right)$.

(3) For every Banach space $Y$ such that $d(X, Y)=1, c \not \subset Y$.

Proof. The implication $(2) \Rightarrow(1)$ follows from Proposition 3.6. We recall that the implication $\neg(3) \Rightarrow \neg(1)$ holds by Theorem 3.2 in [5]. We shall prove that $\neg(2) \Rightarrow \neg(3)$. Let $y_{m}^{*} \in \ell_{1}$ be the $\sigma\left(\ell_{1}, W_{f(m)}\right)$-limit of $\left(e_{n}^{*}\right)$. Put

$$
\widetilde{y_{m}^{*}}=(\underbrace{0, \ldots, 0}_{m-1}, y_{m}^{*}, 0,0, \ldots) \in\left(\sum_{m=1}^{\infty} W_{f^{(m)}}\right)_{c_{0}} \text {. }
$$


Suppose that property $(\diamond)$ does not hold. If there is $y_{m_{0}}^{*} \in S_{\ell_{1}}$, then, as in the proof of the implication $\neg(3) \Rightarrow \neg(5)$ in Proposition 3.1, it is enough to replace the component $W_{f^{\left(m_{0}\right)}}$ in $X=\left(\sum_{m=1}^{\infty} W_{f^{(m)}}\right)_{c_{0}}$ by the hyperplane $W_{f_{\infty, \epsilon}^{\left(m_{0}\right)}}$ with $\epsilon(n)=-\operatorname{sgn}\left(f^{\left(m_{0}\right)}(n+1)\right)$ for all $n \in \mathbb{N}$. Otherwise, there is a subsequence $\left(\widetilde{y_{m_{k}}^{*}}\right)$ of $\left(\widetilde{y_{m}^{*}}\right)$ and $y^{*} \in S_{\ell_{1}}$ such that $\lim _{k \rightarrow \infty} p\left(y^{*}, \widetilde{y_{m_{k}}^{*}}\right)=\lim _{k \rightarrow \infty} p\left(y^{*}, y_{m_{k}}^{*}\right)=0$. Let $f \in S_{\ell_{1}}$ with $f(1)=1 / 2$ be such that $\left(e_{n}^{*}\right)$ is $\sigma\left(\ell_{1}, W_{f}\right)$-convergent to $y^{*}$. We can choose a sequence $\left(f_{0, \epsilon_{k}, \pi_{k}}^{\left(m_{k}\right)}\right)_{k \in \mathbb{N}} \subset S_{\ell_{1}}$, with each $f_{0, \epsilon_{k}, \pi_{k}}^{\left(m_{k}\right)} \in \widetilde{\mathcal{F}_{f^{\left(m_{k}\right)}}}$ satisfying $f_{0, \epsilon_{k}, \pi_{k}}^{\left(m_{k}\right)}(1) \geq 1 / 2$, such that $\lim _{k \rightarrow \infty}\left\|f-f_{0, \epsilon_{k}, \pi_{k}}^{\left(m_{k}\right)}\right\|=0$. Define space $Y$ by replacing each component $W_{f^{\left(m_{k}\right)}}$ of $X=\left(\sum_{m=1}^{\infty} W_{f(m)}\right)_{c_{0}}$ by $W_{f_{0, \epsilon_{k}, \pi_{k}}^{\left(m_{k}\right)}}$ and leaving the others unchanged. By Corollary 2.8, $d(X, Y)=1$. Let $\widetilde{Y}=\left(W_{f} \oplus Y\right)_{\infty}$.

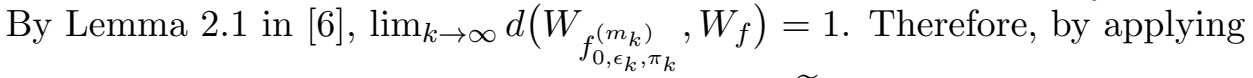
Lemma 3.1 in [14], we easily conclude that $d(Y, \widetilde{Y})=1$. Now we proceed as in the first case.

Remark 3.9. By applying the reasoning from the proof of Proposition 3.8, we easily conclude that property $(\diamond)$ becomes a necessary and sufficient condition for almost stable $w^{*}$-FPP for the whole class of spaces of the form $(X \oplus Y)_{\infty}$, where $X$ is as in Proposition 3.8 and $Y$ is any $\ell_{1}$-predual satisfying (ext $\left.B_{Y^{*}}\right)^{\prime} \subset r B_{\ell_{1}}$ for some $0<r<1$. It is unknown if property $(\diamond)$ characterizes almost stable weak* fixed point property in the general case of $\ell_{1}$-preduals.

As we have already observed, if $X$ is an $\ell_{1}$-predual space satisfying property $(\diamond)$, then for every Banach space $Y$ with $d(X, Y)=1$ we have $Y^{*}=\ell_{1}$ and (ext $\left.B_{Y^{*}}\right)^{\prime} \subset$ int $B_{\ell_{1}}$. However, more can be said:

Proposition 3.10. Property $(\diamond)$ is invariant under the Banach-Mazur distance 1.

Proof. Assume that an $\ell_{1}$-predual $X$ satisfies property $(\diamond)$ and let $Y$ be a Banach space distant by 1 from $X$. Suppose that there exists $y^{*} \in S_{\ell_{1}}$ and a sequence $\left(y_{n}^{*}\right)$ in $\left(\operatorname{ext} B_{Y^{*}}\right)^{\prime}$ such that $\lim _{n \rightarrow \infty} p\left(y_{n}^{*}, y^{*}\right)=0$. By Proposition 2.5, for every $n \in \mathbb{N}$ there exists $x_{n}^{*} \in\left(\text { ext } B_{X^{*}}\right)^{\prime}$ such that $p\left(x_{n}^{*}, y_{n}^{*}\right)<\frac{1}{n}$. Then

$$
p\left(x_{n}^{*}, y^{*}\right) \leq p\left(x_{n}^{*}, y_{n}^{*}\right)+p\left(y_{n}^{*}, y^{*}\right)<\frac{1}{n}+p\left(y_{n}^{*}, y^{*}\right) .
$$

Passing to the limit with $n \rightarrow \infty$, we get a contradiction.

4. Concluding remarks. To tie up ends of this article and [14], we introduce two further definitions. Namely, given a Banach space $X$ having Property $\mathcal{P}$, we will say that Property $\mathcal{P}$ is invariant (or preserved) under the Banach-Mazur distance 1 for the space $X$ if every Banach space 
$Y$ has Property $\mathcal{P}$ whenever $d(X, Y)=1$. On the other hand, we will say that Property $\mathcal{P}$ is not invariant (or not preserved) under the BanachMazur distance 1 for the space $X$ if there exists a Banach space $Y$ such that $d(X, Y)=1$ and $Y$ fails Property $\mathcal{P}$.

For instance, using the notations from [14], the polyhedral properties labelled (pol-iii)-(pol-viii), (pol-K) and their geometric equivalences are invariant under the Banach-Mazur distance 1 for the space $X$ in Example 3.4, $Y$ in Example 3.5, and for all the spaces $X$ satisfying the assumption of Proposition 3.6 (see the implications in the diagram in Section 2 in [14]). Observe that none of these properties is stable for the space $X$ in Example 3.4, even in the restricted setting of $\ell_{1}$-preduals. To see this, consider the hyperplane $W_{\overline{f_{n}}}$ with

$$
\overline{f_{n}}=(1 / 2, \underbrace{-1 /\left[2 \cdot\left(10^{n}-1\right)\right], \ldots,-1 /\left[2 \cdot\left(10^{n}-1\right)\right]}_{10^{n}-1}, 0,0, \ldots), \quad n \in \mathbb{N} .
$$

Then, for each $n \in \mathbb{N}$, consider the isomorphism $\phi_{n}: W_{f_{n}} \rightarrow W_{\overline{f_{n}}}$ defined for every $x=(x(1), x(2), \ldots) \in W_{f_{n}}$ by

$$
\phi_{n}(x)=\left(\frac{10^{n}-1}{10^{n}} x(1), \ldots, \frac{10^{n}-1}{10^{n}} x\left(10^{n}-1\right), x\left(10^{n}\right), x\left(10^{n}+1\right), \ldots\right) .
$$

Next, for every $n \in \mathbb{N}$, define the space $X_{n}$ by replacing the $n$-th component in $X=\left(\sum_{m=1}^{\infty} W_{f_{m}}\right)_{c_{0}}$ by $W_{\overline{f_{n}}}$ and leaving the others unchanged. Then $X_{n}^{*}=\ell_{1}$ and

$$
1 \leq d\left(X_{n}, X\right) \leq d\left(W_{f_{n}}, W_{\overline{f_{n}}}\right) \leq\left\|\phi_{n}\right\|\left\|\phi_{n}^{-1}\right\| \stackrel{n \rightarrow \infty}{\longrightarrow} 1 .
$$

By Proposition 2.1 in [5], $c \subset W_{\overline{f_{n}}} \subset X_{n}$ for every $n \in \mathbb{N}$. This shows that each space $X_{n}$ lacks all these properties, and so they fail to be stable for $X$. Clearly, the same conclusion holds for the space $Y$ in Example 3.5.

On the other hand, all the above-mentioned properties are not invariant under the Banach-Mazur distance 1 for the space $X$ in Example 3.2 in [14]. Also, by Proposition 3.1, the polyhedral properties labelled (pol-iv)-(polviii), (pol-K) and their geometric equivalences are not invariant under the Banach-Mazur distance 1 for the spaces $W_{f}$ discussed in Remark 3.2.

\section{REFERENCES}

[1] Alspach, D. E., A $\ell_{1}$-predual which is not isometric to a quotient of $C(\alpha)$, arXiv:math/9204215v1 [math.FA] 27 Apr. 1992.

[2] Banach, S., Théorie des opérations linéaires, Monografie Matematyczne, Warszawa, 1932.

[3] Cambern, M., On mappings of sequence spaces, Studia Math. 30 (1968), 73-77.

[4] Casini, E., Miglierina, E., Piasecki, E., Hyperplanes in the space of convergent sequences and preduals of $\ell_{1}$, Canad. Math. Bull. 58 (2015), 459-470.

[5] Casini, E., Miglierina, E., Piasecki, Ł., Separable Lindenstrauss spaces whose duals lack the weak $k^{*}$ fixed point property for nonexpansive mappings, Studia Math. 238 (1) (2017), 1-16. 
[6] Casini, E., Miglierina, E., Piasecki, Ł., Popescu, R., Stability constants of the weak fixed point property in the space $\ell_{1}$, J. Math. Anal. Appl. 452 (1) (2017), 673-684.

[7] Casini, E., Miglierina, E., Piasecki, Ł., Popescu, R., Weak* fixed point property in $\ell_{1}$ and polyhedrality in Lindenstrauss spaces, Studia Math. 241 (2) (2018), 159-172.

[8] Casini, E., Miglierina, E., Piasecki, Ł., Veselý, L., Rethinking polyhedrality for Lindenstrauss spaces, Israel J. Math. 216 (2016), 355-369.

[9] Goebel, K., Kirk, W. A., Topics in Metric Fixed Point Theory, Cambridge Univ. Press, Cambridge, 1990.

[10] Japón-Pineda, M. A., Prus, S., Fixed point property for general topologies in some Banach spaces, Bull. Austral. Math. Soc. 70 (2004), 229-244.

[11] Michael, E., Pełczyński, A., Separable Banach spaces which admit $l_{n}^{\infty}$ approximations, Israel J. Math. 4 (1966), 189-198.

[12] Lazar, A. J., Lindenstrauss, J., On Banach spaces whose duals are $L_{1}$ spaces, Israel J. Math. 4 (1966), 205-207.

[13] Pełczyński, A., in collaboration with Bessaga, Cz., Some aspects of the present theory of Banach spaces, in: Stefan Banach Oeuvres. Vol. II, PWN, Warszawa, 1979, 221302.

[14] Piasecki, Ł., On Banach space properties that are not invariant under the BanachMazur distance 1, J. Math. Anal. Appl. 467 (2018), 1129-1147.

\author{
Łukasz Piasecki \\ Institute of Mathematics \\ Maria Curie-Skłodowska University \\ pl. M. Curie-Skłodowskiej 1 \\ 20-031 Lublin \\ Poland \\ e-mail: piasecki@hektor.umcs. lublin.pl
}

Received July 31, 2018 